\title{
A Variable-Temperature Continuous-Flow Liquid-Helium Cryostat Inside a (Scanning) Transmission Electron Microscope
}

\author{
Felix Börrnert ${ }^{1,2}$, Alexander Horst ${ }^{2}$, Michael A. Krzyzowski ${ }^{3}$, and Bernd Büchner ${ }^{2}$ \\ 1. Materialwissenschaftliche Elektronenmikroskopie, Universität Ulm, Albert-Einstein-Allee 11, 89081 \\ Ulm, Germany. \\ 2. IFW Dresden, PF 2701 16, 01171 Dresden, Germany. \\ 3. CryoVac GmbH \& Co. KG, Heuserweg 14, 53842 Troisdorf, Germany.
}

The progress in (scanning) transmission electron microscopy development had led to an unprecedented knowledge of the microscopic structure of functional materials at the atomic level. Additionally, although not widely used yet, electron holography is capable to map the electric and magnetic potential distributions at the sub-nanometer scale. This opens a route to investigate the phase structures of electronic and magnetic phenomena in condensed matter at a microscopic level. Many of the most interesting solid state phenomena occur at low temperatures only. Nevertheless, low temperature studies inside a (scanning) transmission electron microscope ((S)TEM) are extremely challenging because of the much restricted size and accessibility of the sample space. Up to date, there are no cryo-(S)TEMs or special sample holders that are capable to cool a sample controllably to any but its base temperature below room temperature.

Recently, we introduced a concept for a dedicated in-situ (S)TEM for flexible multi-stimuli experimental setups with the capabilities of holographic recording and scanning electron microscopy type imaging. A central part was a large sample chamber with multiple ports. With a prototype instrument, we demonstrated a maximum resolving power of about $1 \mathrm{~nm}$ in conventional imaging mode and substantially better than $5 \mathrm{~nm}$ in scanning mode while providing an effectively usable pole piece gap of $70 \mathrm{~mm}$ [1].

Here, we report about the state of the first major plug-in fitted into the prototype in-situ (S)TEM: A variable-temperature liquid-helium continuous-flow cryostat for nanometer resolved imaging and diffraction at controlled temperatures between $10 \mathrm{~K}$ and $300 \mathrm{~K}$. Arbitrary temperatures in the offered range can be installed and held stable by a heating in the sample mount with the help of a PID controller. The cryostat has two operation modes, one with two cooled radiation shields for temperatures below $10 \mathrm{~K}$ and one without the shields for free sample access from outside the cryostat at temperatures down to $20 \mathrm{~K}$. Sample drift due to negative thermal expansion is reduced by a circular cooled sample mount and a flexible copper strand to the cold finger. The design of a continuous flow cryostat with a low consumption rate offers a long working time at low temperature while sucking helium from a 1001 vessel. Additionally, the cryostat offers four cooled terminals for fixed electrical contacts and is prepared for a future incorporation of two mobile electrical probes.

Examples of experiments now possible with this new setup are the mapping of the phase structure of different electronic and magnetic phenomena, like charge density waves and Skyrmions [2].

\section{References:}

[1] F Börrnert et al, Ultramicroscopy 151 (2015), p. 31.

[2] The authors thank S. Leger for technical assistance. 

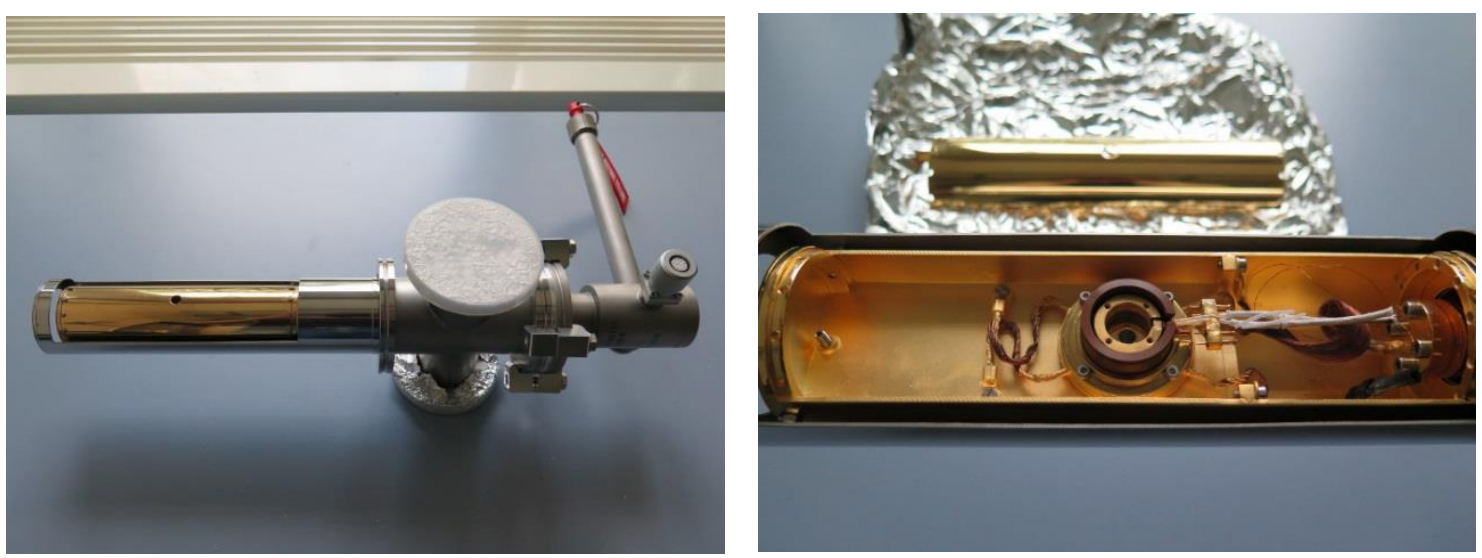

Figure 1. Photographs of the cryostat as delivered, left-with radiation shields mounted and rightopened with a view on the central sample position.
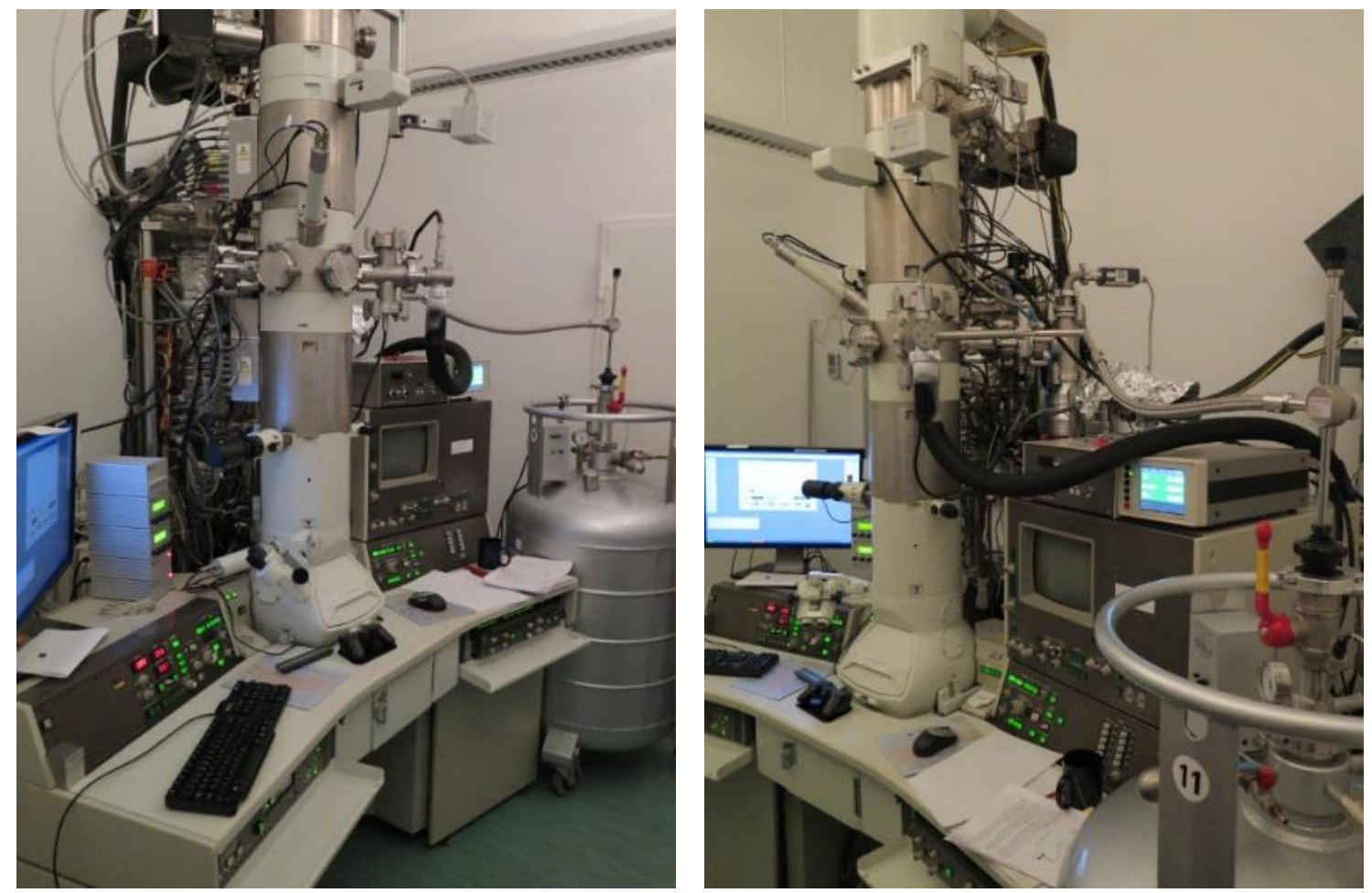

Figure 2. Photographs of the prototype in-situ (S)TEM with the cryostat inserted while cooling. 